\title{
QCD Parton Dynamics, 30 Years later
}

\author{
Yu.L. Dokshitzer \\ LPTHE, Universities of Paris VI-VII and CNRS, Paris, France; \\ St. Petersburg Nuclear Physics Institute, Gatchina, Russia
}

\begin{abstract}
To translate poetry is impossible. But the original is "impossible" too. Then the purpose of a translation is to discover the impossibility of the original. This maxim, belonging to a contemporary poet and philosopher, applies to theoretical physics which attempts to penetrate into the sense of beauty that Einshtein's God indisputably possesses.
\end{abstract}

\section{Introduction}

Physical formulae have reasons. Some are simple and lie on a surface, some may be profound and not even visible at a first examination. Obviously, a formula may not contain a sum of the electric tension and the current, $V+I$. For less obvious (though not less general) reasons, a scattering amplitude may not contain a step-function $\theta$ (causality), or there can be no strong Wan der Waals force between hadrons (relativistic invariance, cross-channel unitarity).

Physical formulae also know to smile. (Recall an ironic $\pi^{2}-9$ factor in the positronium decay width.) A repetitive smile turns into a laughter: the combination $\left(\frac{67}{18}-\frac{\pi^{2}}{6}\right) N_{c}-\frac{5}{9} n_{f}$ that persistently appeared in second loop corrections to DIS structure functions, to jet shape distributions and jet cross sections, in Regge trajectories of quarks and gluon, etc., etc., for a good many year laughs at theorists who got stuck with the MS-bar QCD coupling.

At some point formulae smile no more: they cry for help. This is the case of a body of the third loop corrections to various key QCD quantities that has recently become available as a result of the completion of a breakthrough programme of high order QCD calculations carried out by Moch, Vermaseren and Vogt.

The matrix of the leading order (one loop) anomalous dimensions fitted onto two lines; the second loop result occupied about one page; the volume of the third loop formulae is more natural to measure in (tens of) kilobytes rather than in the number of pages.

Is such a monstrous complexity inevitable? Could it be reasonably reduced? The answer I believe is no, and yes (in this order). I would like to discuss with you two ideas that in my opinion deserve to be explored.

The first idea which I will refer to as "innovative bookkeeping" exploits a hidden symmetry between space- and time-like parton evolution known under the name of the GribovLipatov reciprocity (GLR). It constitutes a reformulation of the parton dynamics in terms of a new evolution equation whose evolution kernel respects GLR in all orders. In this alternative scheme for organising the perturbative expansion, the anomalous dimension is related to the evolution kernel by a non-linear equation. Within this scheme a significant (if not dominant) part of the higher loop corrections to the anomalous dimension turns out to be inherited from the lower orders, thus reducing the complexity of the answer.

The second part of the project is more ambitious. The aim is to understand the major part of the evolution kernel exactly, in all orders of the perturbation theory. It is based on two observations, one physical and another technical. The physical observation is that an essential part of the gluon dynamics is actually classical, according to the well known (but often under-appreciated) Low theorem. "Classical" does not mean "simple". However, it 
has a good chance to be solvable. This belief is based on the second (technical) observation that the QCD shares gluons with super-symmetric Yang-Mills theories and, in particular, with the maximally supersymmetric $\mathcal{N}=4 \mathrm{SYM}$ quantum field theory which is likely to be fully integrable, that is, solvable.

\section{Innovative bookkeeping}

In the standard approach, the anomalous dimensions $\gamma(N)$ are equated with the (Mellin transformed, $x \rightarrow N$ ) parton splitting functions $P(x)$; they are different for the space-like (DIS) and time-like evolution $\left(e^{+} e^{-}\right)$; the "clever evolution variables" are different too.

In the new approach, one disconnects the splitting functions from the anomalous dimensions. By organising the parton evolution picture in both channels on the base of the common evolution variable (parton fluctuation time), the new "Hamiltonian" describing the dynamics of partons - the evolution kernel - turns out to be identical for space- and time-like cascades (the Gribov-Lipatov reciprocity relation holds in all orders).

\subsection{QCD partons and perturbation theory}

The universal nature of the parton dynamics goes under the name of factorisation of collinear ("mass") singularities. Physically, it is due to the fact that quark-gluon multiplication processes happen at much larger space-time distances than the hard interaction itself. It is this separation that makes possible the description of quark-gluon cascades in terms of independent classical parton splitting processes. They succeed one another in a cleverly chosen evolution time, $t \sim \ln Q^{2}$, whose flow "counts" basic parton splittings that occur at well separated, strongly ordered space-time scales. The perturbative structure of the cross section of a given process $i$ characterised by the hardness scale $Q^{2}$ can be cast, symbolically, as a product (convolution) of three factors:

$$
\sigma_{h}^{(p)}\left(\ln Q^{2}\right) \propto C^{(p)}\left[\alpha_{s}(t)\right] \otimes \exp \left(\int_{t_{0}}^{t} d \tau P\left[\alpha_{s}(\tau)\right]\right) \otimes w_{h}\left(t_{0}\right), \quad t \sim \ln Q^{2} .
$$

Here the functions $C\left[\alpha_{s}\right]$ (hard cross section; coefficient function) and $P\left[\alpha_{s}\right]$ (parton evolution; anomalous dimension matrix) are perturbative objets analysed in terms of the $\alpha_{s}$ expansion. The last factor $w_{h}$ embeds non-perturbative information about parton structure of the participating hadron(s) $h$, be it a target hadron in the initial state (parton distribution) or a hadron triggered in the final state (fragmentation function).

A borderline between perturbative and non-perturbative ingredients in (1) is fictitious; it is set arbitrarily by choosing the launching hardness scale $t_{0} \sim \ln Q_{0}^{2}$. This is however not the only arbitrariness present in the representation (1). Namely, beyond the leading approximation (one loop; $P=\mathcal{O}\left(\alpha_{s}\right)$ ), the separation between the $C$ and $\exp (P)$ factors becomes "scheme" dependent. Here one talks about factorisation scheme dependence. Another negotiable object is the expansion parameter $\alpha_{s}$ itself whose definition depends on the ultraviolet renormalisation procedure (renormalisation scheme dependence). The so-called MS-bar scheme based on the precisely prescribed procedure of eliminating ultraviolet divergences, based on the dimensional regularisation won the day as the best suited scheme for carrying out laborious high order calculations. It is the MS-bar scheme in which the parton "Hamiltonian" $P$ was recently calculated in the next-to-next-to-leading accuracy, $\alpha_{s}^{3}[2,3]$. 
Formally speaking, the physical answer does not depend on which schemes (factorisation, renormalisation) one chooses to construct the expansion. There is a big "if" however which renders this motto meaningless. It would have been the case, and consolation, if we had hold of the full perturbative expansion of the answer. But this goal is not only technically unachievable. More importantly, it is actually useless. The perturbative QFT expansions are asymptotic series. This means that starting from some order, $n>n_{\text {crit }}=\operatorname{const}_{(p)} / \alpha_{s}$, the series for any observable $(p)$ inevitably goes haywire and ceases to represent the answer. For QED where $n_{\text {crit }} \sim 100$ this was an academic problem. In QCD on the contrary the best hope the perturbative expansion may offer is a reasonable numerical estimate based on the first few orders of the perturbation theory (whose intrinsic uncertainty can often be linked with genuine non-perturbative effects). This being understood, it becomes legitimate, and mandatory, to play with perturbative series trying to recast a formal $\alpha_{s}$ expansion in the most relevant way, the closest to the physics of the problem.

\subsection{Relating DIS and $e^{+} e^{-}$}

No surprise, the space- and time-like parton cascades are intimately related. In the DIS case the large virtual momentum $q$ transferred from the incident lepton to the target nucleon with momentum $P$ is space-like, $q^{2}<0$. The inelasticity of the process is conveniently characterised by the Bjorken variable $x_{B}=-q^{2} / 2(P q)$. On the other side, inclusive fragmentation of an $e^{+} e^{-}$pair with the total momentum $q$ (large positive invariant mass squared $q^{2}$ ) into a final state hadron with momentum $P$ is characterised by the Feynman variable $x_{F}=2(P q) / q^{2}$ (hadron energy fraction in the $e^{+} e^{-}$cms.). The fact that Bjorken and Feynman variables are indicated by the same letter is certainly not accidental. In both channels $0 \leq x \leq 1$ though these variables are actually reciprocal, $x_{F} \Longleftrightarrow 1 / x_{B}$, rather than identical:

$$
x_{B}=\frac{-q^{2}}{2(P q)}, \quad x_{F}=\frac{2(P q)}{q^{2}} .
$$

One $x$ becomes the inverse of the other after the crossing operation $P_{\mu} \rightarrow-P_{\mu}$. Apart from the difference in the hadron momentum $P$ belonging to the initial state in the DIS and final state in the $e^{+} e^{-}$case, the Feynman diagrams for the two processes are just the same. In particular, mass singularities that emerge when some parton momentum become collinear to $P$ are therefore also the same. That is why, for the two processes a similar parton interpretation emerges in terms of QCD evolution equations, and the space- and time-like evolution anomalous dimensions turn out to be related.

Drell-Levy-Yan relation [4] follows directly from the comparison of the structure of Feynman diagrams in space- and time-like channels. It states that the $e^{+} e^{-}$splitting functions can be obtained from those of DIS by replacing $x_{B} \rightarrow 1 / x_{F}$ (modulo a kinematical factor):

$$
P_{B A}^{(T)}(x)=(-1)^{2\left(J_{A}-J_{B}\right)+1} x \cdot P_{A B}^{(S)}\left(x^{-1}\right)
$$

(with $J_{A}$ the spin of the parton $A$ ). Beyond the leading logarithmic approximation (LLA) $[5,6,7]$, the DLY relation (3) was being used to actually determine the time-like splitting functions from their space-like counterparts. True in any QFT, ${ }^{\mathrm{a}}$ it reflects the crossing and allows one to link the cross sections in the two channels by analytic continuation from $x<1$

${ }^{\mathrm{a}}$ when applied to physical cross sections, not necessarily to scheme dependent anomalous dimensions [8] 
to $x>1$. So doing, one obtains the time-like splitting function, $P^{(T)}(x)$, by analytically continuing the function $P^{(S)}(x)$ into the unphysical region $x>1$, and then replacing $x \rightarrow$ $1 / x<1$. The continuation path crosses the singular point $x=1$ which needs a special care to be taken in defining certain complex logarithms in "arithmetic" sense, $\ln (1-x) \Longrightarrow$ $|\ln (1-x)|$, see $[9,7]$; beyond the first loop, see $[10,8]$ and references therein.

Gribov-Lipatov relation [5] applies in the physical regions of both channels, $x \leq 1$, and states simply that the splitting functions are identical, although their arguments are actually given by the different expressions (2):

$$
P_{B A}^{(T)}\left(x_{\text {Feynman }}\right)=P_{B A}^{(S)}\left(x_{\text {Bjorken }}\right) ; \quad x_{B}=\frac{-q^{2}}{2 p q}, \quad x_{F}=\frac{2 p q}{q^{2}} .
$$

Combined with the DLY relation (3), it becomes the Gribov-Lipatov reciprocity (GLR):

$$
P_{B A}(x)=\mp x \cdot P_{A B}\left(x^{-1}\right) .
$$

True in the leading logarithmic approximation, the Gribov-Lipatov reciprocity (4) was found to be broken beyond the first loop [11]. But why?

\subsection{Long live parton fluctuation time!}

It is instructive to look more carefully into the origin of logarithmically enhanced contributions to the DIS cross section. Introducing two light-like vectors $p_{1}^{\mu}$ and $p_{2}^{\mu}$ one can write down the Sudakov (light-cone) decomposition of momenta:

$$
k^{\mu}=\beta p_{1}^{\mu}+\alpha p_{2}^{\mu}+k_{\perp}^{\mu}, \quad k^{2}=\alpha \beta s-\mathbf{k}_{\perp}^{2} \quad\left(s=2 P q,\left(k_{\perp}^{\mu}\right)^{2}=-\mathbf{k}_{\perp}^{2}\right) .
$$

Then, for $k_{1}^{\mu}+k_{2}^{\mu}+k_{3}^{\mu}=0$ it is straightforward to derive the identity

$$
\frac{k_{1}^{2}}{\beta_{1}}+\frac{k_{2}^{2}}{\beta_{2}}+\frac{k_{3}^{2}}{\beta_{3}}=\frac{\beta_{1} \beta_{2}}{\beta_{3}}\left(\frac{\mathbf{k}_{\perp 1}}{\beta_{1}}-\frac{\mathbf{k}_{\perp 2}}{\beta_{2}}\right)^{2} .
$$

Let us now apply this general relation to the parton splitting that involves a space-like parton $A$ decaying into $B+C$. Choosing for the sake of simplicity the direction of $p_{1}$ such

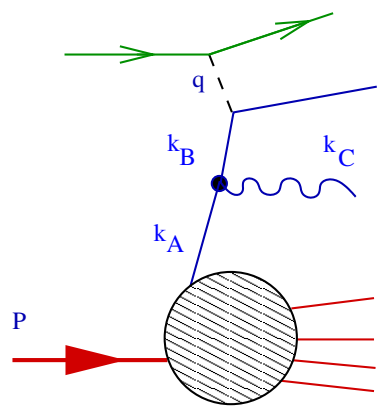

that $\mathbf{k}_{\perp A}=0$ (so that $\mathbf{k}_{\perp B}=-\mathbf{k}_{\perp C} \equiv \mathbf{k}_{\perp}$ is the relative transverse momentum in the splitting) the relation (5) applied to our basic space-like splitting $A \rightarrow B[z]+C[1-z]$ gives

$$
\frac{-k_{B}^{2}}{z}=\frac{-k_{A}^{2}}{1}+\frac{k_{C}^{2}}{1-z}+\frac{\mathbf{k}_{\perp}^{2}}{z(1-z)}
$$

where $z$ is the longitudinal momentum fraction - the ratio of the Sudakov light-cone variables $\beta$. Since the 4 -momenta of $A$ and $B$ are space-like, all terms in (6) are positive.

$B$ being an intermediate virtual state, $k_{B}^{2}$ enters in the Feynman denominators in the matrix element. The collinear-log contribution arises upon the $k_{\perp}^{2}$ integration over the region where the last term dominates in the r.h.s. of (6), that is from the region

$$
\frac{\left|k_{B}^{2}\right|}{z} \simeq \frac{\mathbf{k}_{\perp}^{2}}{z(1-z)} \gg \frac{\left|k_{A}^{2}\right|}{1}, \frac{k_{C}^{2}}{1-z} .
$$


The physical origin of this strong inequality becomes transparent in terms of lifetimes of virtual states $\left(p_{1}^{\mu} \simeq P^{\mu}, p_{2}^{\mu}=q^{\mu}+x P^{\mu}\right)$

$$
\frac{\beta_{i} P}{\left|k_{i}^{2}\right|} \simeq \frac{k_{i}^{0}}{\left|k_{i}^{2}\right|}=\tau_{i}
$$

namely $\tau_{B} \ll \tau_{A}, \tau_{C}$. This shows that the LLA contributions originate from the sequence of branchings well separated in the fluctuation time (7). Invoking the local-scattering analogy (recall $A \rightarrow C$ on the "external field" $B$ ), we can say that the classical picture naturally implies "fast scattering": probing time $\tau_{B}$ much smaller than the proper lifetimes of the "target" before $\left(\tau_{A}\right)$ and after the scattering occurs $\left(\tau_{C}\right)$.

In the DIS kinematics the evolution goes from the proton side and, on the way towards the virtual probe $Q^{2}$, parton fluctuations become successively shorter-lived (the "probe" is faster than the fluctuation time of the "target"). Assembling a "ladder" of successive parton splittings we get the $n^{\text {th }}$-order LLA contribution $\left(\alpha_{s} \ln Q^{2}\right)^{n}$ to come from timeordered kinematics

$$
\frac{P}{\mu^{2}} \gg \tau_{1} \gg \tau_{2} \gg \ldots \gg \tau_{n} \gg \frac{x P}{-q^{2}} ; \quad x=x_{\text {Bjorken }} \equiv \frac{-q^{2}}{2 P q} .
$$

In the case of a time-like evolution of a jet produced, e.g., In the crossing channel, $e^{+} e^{-} \rightarrow$ $q \bar{q} \rightarrow h(x)+X$, the process starts from a large scale $q^{2}$ (cms annihilation energy) and results in triggering a final particle $h$ with momentum $P$. Here the order of events is opposite: a parton of the generation $(i+1)$ lives longer than its parent $(i)$ :

$$
\frac{P}{x q^{2}} \ll \tau_{1} \ll \tau_{2} \ll \ldots \ll \tau_{n} \ll \frac{P}{\mu^{2}} ; \quad x=x_{\text {Feynman }} \equiv \frac{2 P q}{q^{2}},
$$

where we have used that the energy of the initial quark stemming form the $\gamma^{*} \rightarrow q \bar{q}$ vertex is $q_{0} / 2=P / x_{F}$. Comparing the two sequences (8) we see that the reciprocity $x \rightarrow x^{-1}$ is present in the ordering of successive fluctuation lifetimes. So, why does the Gribov-Lipatov relation break up in higher orders? The answer is quite simple: it is because we never followed the fluctuation time ordering ${ }^{\mathrm{b}}$ for constructing the anomalous dimensions. And for a good reason it seemed.

\subsection{Coherence}

Beyond the first loop it starts to matter how one orders successive parton splittings, that is, what one chooses for the parton evolution time $t \sim \ln Q^{2}$.

At the LLA level, it does not make sense to argue which of possible "evolution times", $\ln \left(k^{2} / \beta\right), \ln k^{2}, \ln \mathbf{k}_{\perp}^{2}$ or alike, does a better job: the options differ by subleading terms $\mathcal{O}\left(\alpha_{s}\right)$, negligible as compared to $\alpha_{s} \ln Q \sim 1$. However, when one studies the kinematical region of numerically small $x$, the next-to-LLA mismatch contributions amount to

$$
\alpha_{s} \ln ^{2} \frac{\beta_{i+1}}{\beta_{i}}=\alpha_{s} \ln ^{2} z \quad \Longrightarrow \quad\left(\alpha_{s} \ln ^{2} x\right)^{n}
$$

\footnotetext{
${ }^{\mathrm{b}}$ markedly, with an exception of the study of QCD coherent states within the asymptotic dynamics framework, [12] and references therein
} 
and may become significant. If $\alpha_{s} \ln ^{2} x \sim 1$, they must be taken into account in all orders. In this situation the soft gluon radiation comes onto stage. And here we better be careful: the catch is, for a relatively soft gluon with $z \ll 1$, to be emitted later does not guarantee to be emitted independently. Interfering diagrams with the gluon radiation off harder partons of different generations enter the game, thus endangering the probabilistic parton picture. It was realised quite some time ago that the probabilistic interpretation can be rescued by simply cutting off a definite part of the logarithmic phase space that is formally allowed by the "kinematical" fluctuation time ordering.

In the DIS environment, the transverse momentum ordering proved to be the one that takes care of potentially disturbing corrections (9) in all orders, and in this sense became a preferable choice for constructing the probabilistic scheme for space-like parton cascades (DIS structure functions). On the other hand, in the case of the time-like cascades it turned out to be the relative angle between the offspring partons (rather than the transverse momentum) which had to be kept ordered and decreasing along the evolutionary decay chain, from the hard production vertex towards the registered inclusive hadron:

$$
\begin{aligned}
& d t_{-}=d \ln k_{\perp}^{2} \quad(\text { space-like }), \\
& d t_{+}=d \ln \frac{k_{\perp}^{2}}{\beta^{2}} \quad(\text { time-like }) .
\end{aligned}
$$

(Since $k_{\perp} / \beta P=k_{\perp} / k_{+}=2 \tan (\theta / 2)$, the variable (10b) corresponds indeed to the angular ordering.) What is the difference between the two prescriptions (10) and how do they relate with the fluctuation time ordering discussed above and represented by (8)? For $z \ll 1$ we have $\left|k^{2}\right| \simeq k_{\perp}^{2}$ and the comparison goes as follows

$$
\text { DIS }\left\{\begin{array}{cc}
\text { time ordering: } & \tau_{i}=\frac{\beta_{i} P}{k_{\perp i}^{2}}>\tau_{i+1}=\frac{\beta_{i+1} P}{k_{\perp i+1}^{2}}, \\
k_{\perp} \text { ordering: } & k_{\perp i}<k_{\perp i+1} ; \\
\text { mismatch } \Longrightarrow & z \cdot k_{\perp i}^{2}<k_{\perp i+1}^{2}<k_{\perp i}^{2},
\end{array}\right.
$$

while for the time-like cascades

$$
e^{+} e^{-}\left\{\begin{aligned}
\text { time ordering: } & \tau_{i}=\frac{\beta_{i} P}{k_{\perp i}^{2}}<\tau_{i+1}=\frac{\beta_{i+1} P}{k_{\perp i+1}^{2}}, \\
\text { angular ordering: } & \theta_{i}=\frac{k_{\perp i}}{\beta_{i} P}>\theta_{i+1}=\frac{k_{\perp i+1}}{\beta_{i+1} P} \\
\text { mismatch } \Longrightarrow & \theta_{i}^{2}<\theta_{i+1}^{2}<\frac{\theta_{i}^{2}}{z}
\end{aligned}\right.
$$

We conclude that in both cases the fluctuation time ordering turns out to be more liberal. The destructive soft gluon interference suppresses the kinematical "mismatch" regions. Physically, the disappearance of the region (11a) in the space-like kinematics is related to the general phenomenon of the vanishing of the forward inelastic diffraction (Gribov, late 1960s); the disappearance of the angular disordered region (11b) can be traced back to the so-called Chudakov effect in the cosmic ray physics.

\subsection{Rescuing Gribov-Lipatov reciprocity}

Thus, the choice of the variables $(10)$ is a clever dynamical move that takes into full consideration soft gluon coherence and prevents explosively large terms (9) from appearing in higher 
loop anomalous dimensions. Not only is the fluctuation time ordering wrong both in spaceand time-like kinematics, it happens to be equally, symmetrically wrong: the $\tau$-ordering positions itself just in the middle between the two "clever" ones:

$$
k_{\perp}^{2} \Longrightarrow \frac{k_{\perp}^{2}}{z} \Longrightarrow \frac{k_{\perp}^{2}}{z^{2}} .
$$

What if we decide to play a fool and stubbornly stick to the "wrong" $\tau$-ordering?

Combining (8) and (7) we get the upper limits of virtuality integrals to be

$$
\left.\begin{array}{ll}
\text { DIS : } & \left|k_{i}^{2}\right| \ll \frac{\beta_{i}}{\beta_{i+1}}\left|k_{i+1}^{2}\right|=z^{-1} \cdot\left|k_{i+1}^{2}\right| \\
e^{+} e^{-}: & k_{i+1}^{2} \ll \frac{\beta_{i+1}}{\beta_{i}} k_{i}^{2}=z \cdot k_{i}^{2}
\end{array}\right\} \quad z=\frac{\beta_{i+1}}{\beta_{i}} \leq 1 .
$$

Different placing of the $z$ factor causes (beyond the first loop) a violation of the GribovLipatov reciprocity. Moreover, it is likely to be the one and only source of this breaking!

Let us probe this idea. Choosing $\kappa^{2}=\left|k^{2}\right|$ as an integration variable and assembling parton evolution sequences, for the probability $D\left(x, Q^{2}\right)$ to find a parton with virtuality integrated up to a given $Q^{2}$ we obtain (omitting a trivial Born term) $[13,14]$

$$
D\left(x, Q^{2}\right)=\int_{x}^{1} \frac{d z}{z} \int^{Q^{2}} \frac{d \kappa^{2}}{\kappa^{2}} P\left[z, \alpha_{s}\right] D\left(\frac{x}{z}, z^{\sigma} \kappa^{2}\right) ; \quad \sigma= \pm 1 \text { for the } \mathrm{T} / \mathrm{S} \text { channel. }
$$

The second argument of the $D$ function on the r.h.s. of the equation follows from (12). In terms of the Mellin moment representation of parton distributions and splitting functions,

$$
D_{N}\left(Q^{2}\right)=\int_{0}^{1} \frac{d x}{x} x^{N} D\left(x, Q^{2}\right), \quad \mathcal{P}\left(N, \alpha_{s}\right)=\int_{0}^{1} d z z^{N-1} P\left[z, \alpha_{s}\right],
$$

by dropping in (13) the $z^{\sigma}$ factor, one would obtain

$$
\partial_{\ln Q^{2}} D_{N}\left(Q^{2}\right) \equiv \gamma\left(N, \alpha_{s}\right) D_{N}\left(Q^{2}\right)=\int_{0}^{1} \frac{d z}{z} z^{N} P\left[z, \alpha_{s}\right] D_{N}\left(Q^{2}\right)=\mathcal{P}\left(N, \alpha_{s}\right) D_{N}\left(Q^{2}\right),
$$

which equates the anomalous dimension with the Mellin image of the corresponding splitting function: $\gamma_{-} \equiv \mathcal{P}^{(S)}$ and $\gamma_{+} \equiv \mathcal{P}^{(T)}$. The presence of the factor $z^{\sigma}$ in the second argument of (13) makes the evolution equation non-local in longitudinal $(x)$ and transverse variables $\left(Q^{2}\right)$ and breaks the identification between the splitting functions and anomalous dimensions. What it offers instead is a link between the two channels by means of the universal reciprocity respecting parton splitting matrix $\mathcal{P}$, the same for $\mathrm{T}$ and $\mathrm{S}$ evolutions. In spite of the fact that the new evolution kernel in (13) does not correspond to any clever choice of the evolution variable (explosive $\alpha_{s} \ln ^{2} x$ terms being present in both channels), this universality can be exploited to relate the DIS and $e^{+} e^{-}$anomalous dimensions.

Differentiating (13) and taking Mellin moments of the differential equation

$$
\partial_{\ln Q^{2}} D\left(x, Q^{2}\right)=\int_{x}^{1} \frac{d z}{z} P\left[z, \alpha_{s}\right] D\left(\frac{x}{z}, z^{\sigma} Q^{2}\right),
$$


one obtains

$$
\gamma_{\sigma}(N) D_{N}=\int_{0}^{1} \frac{d z}{z} z^{N} P\left[z, \alpha_{s}\right] z^{\sigma \partial_{\ln Q^{2}} D_{N}} .
$$

This integral can be formally evaluated using the Taylor expansion trick,

$$
\gamma_{\sigma}(N)=\left(D_{N}\right)^{-1} \mathcal{P}\left(N+\sigma \partial_{\ln Q^{2}}\right) D_{N},
$$

expressing the anomalous dimension through the Mellin image of the splitting function that depends on the differential operator as an argument, $N \rightarrow N+\sigma \partial_{\ln Q^{2}}$. The derivative acts upon $D_{N}=D_{N}\left(Q^{2}\right)$ producing, by definition, $\gamma(N) \cdot D_{N}$. In high orders it will also act on the running coupling the anomalous dimension depends on, $\gamma=\gamma\left(N, \alpha_{s}\right)$. The latter action gives rise to terms proportional to the $\beta$-function. Such terms are scheme dependent as they can be reshuffled between the exponent and the coefficient function $C\left[\alpha_{s}\right]$ in (1). Neglecting for the time being these contributions by treating $\alpha_{s}$ as a constant, (14) reduces to a compact functional equation

$$
\gamma_{\sigma}(N)=\mathcal{P}\left(N+\sigma \gamma_{\sigma}(N)\right) .
$$

Since $\gamma=\mathcal{O}\left(\alpha_{s}\right)$, we can expand the argument of the splitting function perturbatively,

$$
\gamma_{\sigma}=\mathcal{P}+\dot{\mathcal{P}} \cdot \sigma \gamma_{\sigma}+\frac{1}{2} \ddot{\mathcal{P}} \cdot \gamma_{\sigma}^{2}+\mathcal{O}\left(\beta\left(\alpha_{s}\right)\right)+\mathcal{O}\left(\alpha_{s}^{4}\right)
$$

where each dot marks the derivative over the moment $N$. Solving (16a) iteratively,

$$
\gamma_{\sigma}=\mathcal{P}+\sigma \mathcal{P} \dot{\mathcal{P}}+\left[\mathcal{P} \dot{\mathcal{P}}^{2}+\frac{1}{2} \mathcal{P}^{2} \ddot{\mathcal{P}}\right]+\ldots,
$$

and restricting to the first loop, $\mathcal{P}=\alpha_{s} P^{(1)}$ (with $P^{(1)}$ the Mellin image of the good old LLA splitting functions) gives

$$
\gamma_{\sigma}=\alpha_{s} P^{(1)}+\alpha_{s}^{2} \sigma P^{(1)} \dot{P}^{(1)}+\ldots
$$

Applied to the non-singlet quark evolution, the second term on the r.h.s. of (17) generates the GLR breaking observed by Curci, Furmanski \& Petronzio in the second loop [11]. This shows that the GLR violation is not a dynamical higher order effect but is inherited from the previous $\operatorname{loop}(\mathrm{s})$ via a non-linear relation. In three loops, the GLR breaking predicted by (14) was verified in [15].

The same structure of the GLR violation holds for the gluon $\rightarrow$ gluon evolution as well. Strictly speaking, this is true only for two colour structures that emerge in the second loop anomalous dimensions $\mathcal{P}_{g g}^{(2)}$ namely, $C_{A}^{2}$ and $C_{A} C_{F}$. The third one, $2 n_{f} T_{R} C_{F}$, corresponds to the $g \rightarrow q(\bar{q}) \rightarrow g$ two-step transition that mixes gluon and quark states. This colour factor is also present in the singlet quark evolution, $q \rightarrow g \rightarrow q$, described by the two-loop anomalous dimensions $\mathcal{P}_{q q, s}^{(2)}$. As a result, the structure of the GLR breaking in this specific colour structure turned out to be more involved though natural [13]:

$$
\frac{1}{2}\left[\mathcal{P}_{q q, s}^{(2, T)}-\mathcal{P}_{g g}^{(2, S)}\right] \Longrightarrow \mathcal{P}_{g q}^{(1)} \dot{\mathcal{P}}_{q g}^{(1)}, \quad \frac{1}{2}\left[\mathcal{P}_{g g}^{(2, T)}-\mathcal{P}_{q q, s}^{(2, S)}\right] \Longrightarrow \mathcal{P}_{q g}^{(1)} \dot{\mathcal{P}}_{g q}^{(1)}
$$

The analysis of non-diagonal parton transitions is more difficult since here the scheme dependence is more pronounced. Stratmann and Vogelsang have addressed this issue in [10] where a detailed discussion was given of a possibility to rescue GLR in two loops in terms of factorisation scheme transformation. The problem should be further pursued. 


\subsection{RREE applications}

An important aspect of the reciprocity respecting evolution equation (RREE) is the dual nature of the expansion (16): it is obviously a series in the coupling $\left(\mathcal{P} \propto \alpha_{s}\right)$; at the same time, it is an expansion in $N^{-1}$. Indeed, the leading behaviour of the evolution kernel is logarithmic, $\mathcal{P}(N) \propto \alpha_{s} \ln N$, so that each successive term in (16b) acquires a $1 / N$ suppression factor: $\dot{\mathcal{P}} \sim N^{-1}, \ddot{\mathcal{P}} \sim \dot{\mathcal{P}}^{2} \sim N^{-2}$, etc. In the $x$ space, $N^{-1}$ translates into $(1-x)$ - another expansion parameter that becomes small in the quasi-elastic kinematics when the invariant mass of the final state hadron system is much smaller than the hardness scale of the process: $W^{2} \simeq Q^{2}(1-x) / x \ll Q^{2}$.

In the $x \rightarrow 1$ limit (large moments $N)$ the diagonal anomalous dimensions $(q \rightarrow q, g \rightarrow g)$ have a general structure

$$
\gamma(x)=\frac{A x}{(1-x)_{+}}+B \delta(1-x)+C \ln (1-x)+D+\mathcal{O}\left((1-x) \log ^{p}(1-x)\right),
$$

where $A, B, C, D$, are series in $\alpha$ known nowadays to three loops. In particular,

$$
C_{F}^{-1} \cdot \frac{A_{q}}{4 \pi}=C_{A}^{-1} \cdot \frac{A_{g}}{4 \pi} \equiv \alpha_{\text {phys }}
$$

is the physical coupling (cusp anomalous dimension [16]) which determines the radiation intensity of relatively soft gluons $[17,14]$. It universally appears in higher order corrections to observables that are sensitive to soft gluon emission: anomalous dimensions and coefficient functions in the quasi-elastic limit, Sudakov quark and gluon form factors (threshold resummation, the Drell-Yan $K$-factor, distributions of jet event shapes in the near-to-two-jet kinematics, heavy quark fragmentation functions, etc.), in quark and gluon Regge trajectories, even in power suppressed effects in jet shapes and elsewhere.

Soft radiation has a classical nature and is governed by the celebrated Low (LowBurnett-Kroll; LBK) theorem [18]. LBK taught us that both the singular, $(1-x)^{-1}$, and the constant terms, $(1-x)^{0}$, in the photon/gluon emission are universal and contained by the first $(A)$ structure in (18), while the quantum contributions vanish in the $x \rightarrow 1$ limit as $(1-x)$. The terms $C$ and $D$ fall into the gap between the classical and quantum physics. In the leading order, $C=D=0$; beyond the leading order, these structures must be therefore deducible rather than genuine higher order corrections. Indeed, the RREE answers the call: given the physical coupling $A\left(\alpha_{s}\right)$ and the virtual correction term $B\left(\alpha_{s}\right)$, one obtains the all-order relations $C=-\sigma A^{2}[2,19]$ and $D=-\sigma A B+\mathcal{O}(\beta)[19,20]$.

Another interesting thing that the RREE offers is a possibility to link together two puzzling perturbative results that were never thought to be of a common origin. These are: the absence of the $\alpha_{s}^{2}$ and $\alpha_{s}^{3}$ terms in the BFKL anomalous dimension in the DIS problem, and, on the other hand, the phenomenon of the exact angular ordering that was found to miraculously apply down to the next-to-next-to-leading order in $e^{+} e^{-}$parton cascades.

The deep universal nature of the RREE was elucidated by Basso and Korchemsky who have derived (15) from the conformal invariance. In the moment space, the GLR (4) translates into the internal symmetry of the large- $N$ asymptotic series for the anomalous dimensions which relates the terms of even and odd power of $1 / N$ ("parity preserving series"). From this perspective, the validity of the RRE was verified in [20] for various anomalous dimensions that had been calculated to higher orders in different quantum field theories.

A special place in this list is occupied by super-symmetric QFTs, and by the maximally super-symmetric $\mathcal{N}=4$ Yang-Mills model in particular. 


\section{Divide and conquer}

The integrability feature manifests itself in certain sectors of QCD, in specific problems like

- the high energy Regge behaviour in the large $N_{c}$ ('t Hooft) limit [22],

- the spin $\frac{3}{2}$ baryon wave function [23],

- the scale dependence of the maximal helicity multi-gluon operators [24].

In each of these problems (and within the corresponding approximation) one can identify QCD with one or another of its SUSY partner QFTs. The higher the symmetry, the deeper the integrability. From this point of view the $\mathcal{N}=4$ SYM theory is at the top of the pyramid. It is conformally invariant at the quantum level $\left(\beta\left(\alpha_{s}\right) \equiv 0\right)$ and is likely to be fully integrable, via the AdS/CFT correspondence [25].

In $\mathcal{N}=4 \mathrm{SYM}$ all twist-2 operators belong to one super-multiplet. As a result, all five anomalous dimensions (three for unpolarized distributions of gluons, gauginos and scalars, and two polarized ones) after diagonalization get expresses in terms of the unique function — the "universal anomalous dimension" $\gamma_{\text {uni }}(N)$ with shifted arguments. Inspired by the structure of the answer in the first two loops, Kotikov, Lipatov, Onishchenko and Velizhanin (KLOV) have suggested that $\gamma_{\text {uni }}^{(n)}$ at $n$ loops is built of Euler-Zagier harmonic sums of transcedentality $\tau=2 n-1$. This "maximum transcendentality principle" allowed them to predict $\gamma_{\text {uni }}^{(3)}$ by simply picking up from the multi-page three-loop non-singlet QCD anomalous dimension [2] the most complicated terms (the maximal transcendentality ones, $\tau=5$ ) [26].

A half-page long KLOV three-loop anomalous dimension turned out to be generated by a reciprocity respecting evolution kernel that fits on one line [27]. Applied to the four-loop anomalous dimension of twist three single trace operators built of three scalar fields that has been recently calculated by solving numerically the Bethe Ansatz equations in [28, 29], the RREE has demonstrated an even more impressive power of "compactification" [30].

The innovative bookkeeping, when properly developed, may perform a fantastic quest of generating $\gamma_{\text {uni }}$ of $\mathcal{N}=4 \mathrm{SYM}$ in all orders of the perturbative expansion. Now that we learned that in this theory the physical coupling $A$ can be calculated in all orders [31], such a dream does not look too crazy.

Listening attentively, you may catch the leitmotiv of the $\mathcal{N}=4$ SYM song: its dynamics is devoid of quantum effects and looks essentially classical. The features that speak (or rather sing in unison) to this effect are: the integrability, the all-order Parke-Taylor amplitudes, the zero $\beta$-function and, last but not least, the fact that the basic parton Hamiltonian (one-loop $\left.\gamma_{\text {uni }}\right)$ contains but soft classical gluons, in the LBK meaning of the term.

Recall the structure of the elementary QCD gluon emission probabilities:

$$
\begin{aligned}
P_{q \rightarrow q(x)+g} & =\frac{C_{F} \alpha_{s}}{\pi}\left[\frac{x}{1-x}+(1-x) \cdot \frac{1}{2}\right] \\
P_{g \rightarrow g(x)+g} & =\frac{C_{A} \alpha_{s}}{\pi}\left[\frac{x}{1-x}+(1-x) \cdot\left(x+x^{-1}\right)\right] .
\end{aligned}
$$

The first term on the r.h.s. is the universal piece due to LBK "classical gluons"; the second terms depend on the nature of the radiator and are due to "quantum gluons" (clagons and quagons, if you please).

\footnotetext{
${ }^{\mathrm{c}}$ for a review see $[21]$
} 
Clagons (classical field) and guagons (quantum degrees of freedom) have different nature and play complementary rôles in the QCD play. Radiation of the first is infrared singular, $d \omega / \omega$, and its intensity determines the physical coupling; the second are infrared irrelevant, $\omega d \omega$, but it is them who make the coupling run. Clagons are responsible for the double logarithmic radiative effects (quark and gluon form factors, reggeization); small transverse momentum clagons form the "Lund string" (gluers). Quagons are responsible for changing the state of the radiating object ( $P$ and $C$ parity, colour) while clagons do not carry quantum numbers (sic!). Importantly, it is clagons that determine the major part of the QCD anomalous dimensions. ${ }^{\mathrm{d}}$

QCD shares the gluon sector with its SUSY companions, and the $\mathcal{N}=4$ SYM universal anomalous dimension contains but clagons - the contributions of scalars, gauginos and "hard gluons" (quagons) cancel in the anomalous dimension (as they did in the $\beta$-function):

$$
P(x)=\frac{C_{A} \alpha_{s}}{\pi} \frac{x}{1-x}, \quad \gamma_{\text {uni }}(N)=\int_{0}^{1} \frac{d x}{x}\left[x^{N}-1\right] P(x)=\psi(N+1)+\gamma_{E} \equiv S_{1}(N) .
$$

$S_{1}$ is the basic Euler harmonic function - the Mellin image of the classical LBK radiation.

The mystery of the KLOV maximum transcedentality principle may be explained by the fact that the classicality of the $\mathcal{N}=4 \mathrm{SYM}$ dynamics pertains in higher loops: the generalized Euler-Zagier harmonic sums describe re-interaction of classical gluons higher order, while the truly quantum effects never enter the stage.

\section{Conclusions}

The notion of the reciprocity respecting evolution equation (RREE) emerged in an attempt to combine anomalous dimensions of space-like parton distributions and time-like parton fragmentation functions in a single framework. It showed that the complexity of higher loop contributions is, to a large extent, inherited from lower orders. This is especially so for the major part of the QCD anomalous dimensions governed by the "classical" gluon radiation, in the sense of the Low-Burnett-Kroll theorem. I believe there should exist a framework in which the effects of classical gluon fields would be fully generated, in all orders, from the first loop, in the spirit of the LBK wisdom. A joy of accomplishing such an ambitious programme would rightfully match that of the creator of the harmonic functions:

"However sublime are the researches on fluids which we owe to Messrs Bernoulli, Clairaut and d'Alembert, they flow so naturally from my two general formulae that one cannot sufficiently admire this accord of their profound meditations with the simplicity of the principles from which I have drawn my two equations."

Leonard Euler

\footnotetext{
${ }^{\mathrm{d}}$ In a specific example of heavy quark fragmentation functions the innovative bookkeeping was shown to reduce genuine second loop (quantum) corrections to miserable $2 \%$ [14].
} 


\section{References}

[1] Slides: http://indico. cern. ch/contributionDisplay $\cdot$ py? contribId=1\&sessionId=2\&conf Id=9499

[2] S. Moch, J.A.M. Vermaseren and A. Vogt, Nucl. Phys. B688 101 (2004);

[3] A. Vogt, S. Moch and J.A.M. Vermaseren, Nucl. Phys. B691 129 (2004).

[4] S.D. Drell, D.J. Levy and T.M. Yan, Phys. Rev. 1872159 (1969); ibid. D1, 1617 (1970).

[5] V.N. Gribov and L.N. Lipatov, Sov. J. Nucl. Phys. 15, 438 (1972); ibid p. 675; L.N. Lipatov, Sov. J. Nucl. Phys. 2094 (1975).

[6] G. Altarelli and G. Parisi, Nucl. Phys. B126 298 (1977).

[7] Yu.L. Dokshitzer, Sov. Phys. JETP 46641 (1977).

[8] J. Blümlein, V. Ravindran and W.L. van Neerven, Nucl.Phys. B586 349 (2000).

[9] A.P. Bukhvostov, L.N. Lipatov and N.P. Popov, Sov. J. Nucl. Phys. 20287 (1975).

[10] M. Stratmann and W. Vogelsang, Nucl.Phys. B496 41 (1997).

[11] G. Curci, W. Furmanski and R. Petronzio, Nucl. Phys. B175 27 (1980); W. Furmanski and R. Petronzio, Phys. Lett. 97B 437 (1980).

[12] M. Ciafaloni, Phys. Lett. B150 379 (1985); S. Catani, M. Ciafaloni and G. Marchesini, Nucl. Phys. B264 588 (1986).

[13] Yu.L. Dokshitzer, talk at the HERA Workshop, Hamburg, 1993, unpublished.

[14] Yu.L. Dokshitzer, V.A. Khoze and S.I. Troian, Phys. Rev. D53 89 (1996).

[15] A. Mitov, S. Moch and A. Vogt, Phys. Lett. B638 61 (2006).

[16] G.P. Korchemsky, Mod. Phys. Lett. A4 1257 (1989); G.P. Korchemsky and G. Marchesini, Nucl. Phys. B406 225 (1993).

[17] S. Catani, G. Marchesini and B.R. Webber, Nucl. Phys. B349 635 (1991).

[18] F.E. Low, Phys.Rev. 110974 (1958) ; T.H. Burnett and N.M. Kroll, Phys. Rev. Lett. 2086 (1968).

[19] Yu.L. Dokshitzer, G. Marchesini and G.P. Salam, Phys. Lett. B634 504 (2006).

[20] B. Basso and G.P. Korchemsky, Nucl. Phys. B775 1 (2007).

[21] A.V. Belitsky, V.M. Braun, A.S. Gorsky and G.P. Korchemsky, Int. J. Mod. Phys. A 19, 4715 (2004).

[22] L.N. Lipatov, Phys. Lett. B309 394 (1993); JETP Lett. 59596 (1994); L.D. Faddeev and G.P. Korchemsky, Phys. Lett. B342 311 (1995).

[23] V.M. Braun, S.E. Derkachov, G.P. Korchemsky and A.N. Manashov, Nucl. Phys. B 553, 355 (1999) ; A.V. Belitsky, Phys. Lett. B453 59 (1999); Nucl. Phys. B558 259 (1999).

[24] L.N. Lipatov, Evolution equations in QCD, in Perspectives in Hadronic Physics, eds. S. Boffi, C. Ciofi Degli Atti, M. Giannini, World Scientific (Singapore, 1998) p. 413;

J.A. Minahan and K. Zarembo, J. High Ener. Phys. 0303013 (2003); N. Beisert and M. Staudacher, Nucl. Phys. B670 439 (2003).

[25] J.M. Maldacena, Adv. Theor. Math. Phys. 2231 (1998); S.S. Gubser, I.R. Klebanov and A.M. Polyakov, Phys. Lett. B428 105 (1998); E. Witten, Adv. Theor. Math. Phys. 2253 (1998).

[26] A.V. Kotikov, L.N. Lipatov, A.I. Onishchenko and V.N. Velizhanin, Phys. Lett. B595 521 (2004); Erratum ibid. B632 754 (2006)].

[27] Yu.L. Dokshitzer and G. Marchesini, Phys.Lett. B646 189 (2007) .

[28] M. Beccaria, Anomalous dimensions at twist 3 of the sl(2) sector of $N=4 S Y M$, hep-th/0704.3570.

[29] A.V. Kotikov, L.N. Lipatov, A. Rej, M. Staudacher and V.N. Velizhanin, Dressing and Wrapping, hep-th/0704.3586.

[30] M. Beccaria, Yu.L. Dokshitzer and G. Marchesini, Twist 3 of the sl(2) sector of $N=4 S Y M$ and reciprocity respecting evolution, hep-th/0705.2639.

[31] N. Beisert, B. Eden and M. Staudacher, J. Stat. Mech. 0701021 (2007). 